\title{
STRUCTURE FACTOR OF BOSE-CONDENSED SYSTEMS
}

\author{
V. I. Yukalov \\ Bogoliubov Laboratory of Theoretical Physics, \\ Joint Institute for Nuclear Research, Dubna 141980, Russia \\ (Received February 2, 2007; received in final form March 1, 2007)
}

\begin{abstract}
The structure factor for a Bose system with Bose-Einstein condensate is considered in the frame of the self-consistent mean-field approximation. The accomplished analysis demonstrates the principal importance of the following three points: the necessity of preserving the approximation order, the necessity of taking into account anomalous averages, and the necessity of gauge symmetry breaking. If any one of these necessary conditions is not satisfied, calculations yield the appearance of unphysical divergences of the structure factor implying the occurrence of fictitious instability, which contradicts experiments.
\end{abstract}

Key words: Bose-Einstein condensate; structure factor; gauge symmetry breaking; selfconsistent mean-field approximation.

PACS number(s): 03.75.Hh, 03.75Nt, 05.30.Ch, 05.30.Jp, 67.40.Db

\section{INTRODUCTION}

The structure factor is an important quantity characterizing, as follows from its name, the structural properties of the considered system as well as the spectrum of collective excitations. The structure factor can be measured in scattering experiments. It is also related to other directly measurable quantities, such as the isothermal compressibility and sound velocity.

The structure factor of Bose systems has been repeatedly investigated for the case of liquid ${ }^{4} \mathrm{He}$, both theoretically $[1,2]$ and experimentally [3-9]. In recent years, the physics of dilute Bose gases [10] has attracted a great deal of attention (see review articles [11-15]). The structure factor of cold trapped atoms was also measured [16]. Contrary to liquid helium, for which strong interparticle correlations must be accurately taken into account $[2,17]$, dilute gases allow for their description the use of the mean-field approach. Thus, the measured structure factor of dilute trapped ${ }^{87} \mathrm{Rb}$ atoms was found [16] to be in good agreement with the Bogoliubov approximation $[18,19]$. The latter is known to be well justified for weakly nonideal Bose gases, with asymptotically small interactions. When atomic interactions are not asymptotically weak, but rather finite, their theory encounters the notorious Hohenberg-Martin [20] dilemma of conserving versus gapless approaches. This dilemma has recently been resolved by constructing a representative statistical ensemble for Bose systems with a broken gauge symmetry $[21,22]$ and by developing a self-consistent mean-field approximation [23-26].

The aim of the present paper is to consider the structure factor of a dilute Bose-condensed system, in the frame of a self-consistent mean-field approximation [2126 , and to analyze the properties of the structure factor. This approximation, as is shown in Ref. [26], can be employed for both weak and strong atomic interactions. Under the Bose-condensed system, one can keep in mind atomic dilute Bose gases [10-15], molecular systems formed by bound bosons [27-30] and fermions [31-
35], which can be achieved by invoking the Feshbach resonance [36,37], or other effectively bosonic assemblies.

Throughout the paper the system of units is used, where the Planck constant and Boltzmann constant are set to unity, $\hbar \equiv 1, k_{\mathrm{B}} \equiv 1$.

\section{BOSE-CONDENSED SYSTEM}

Phase transitions are known to usually be accompanied by spontaneous symmetry breaking [38]. The appearance of Bose-Einstein condensate is associated with the breaking of global gauge symmetry. The most convenient method of breaking the latter is by means of the Bogoliubov shift $[39,40]$. Then, the field operator is represented in the shifted form

$$
\hat{\psi}(\mathbf{r}, t) \equiv \eta(\mathbf{r}, t)+\psi_{1}(\mathbf{r}, t),
$$

in which $\eta(\mathbf{r}, t)$ is a nonoperator function, defining the condensate wave function, and $\psi_{1}(\mathbf{r}, t)$ is the field operator of uncondensed particles, satisfying the Bose commutation relations. The field variables of condensed and uncondensed particles are orthogonal to each other,

$$
\int \eta^{*}(\mathbf{r}, t) \psi_{1}(\mathbf{r}, t) d \mathbf{r}=0
$$

The condensate function is normalized to the number of condensed particles

$$
N_{0}=\int|\eta(\mathbf{r}, t)|^{2} d \mathbf{r},
$$

while the operator of uncondensed particles satisfies the normalization

$$
N_{1}=\left\langle\hat{N}_{1}\right\rangle
$$

given by statistically averaging the operator

$$
\hat{N}_{1} \equiv \int \psi_{1}^{\dagger}(\mathbf{r}, t) \psi_{1}(\mathbf{r}, t) d \mathbf{r}
$$


for the number of uncondensed particles. The average number of particles in the whole system is $N=N_{0}+N_{1}$. The statistical condition

$$
\left\langle\psi_{1}(\mathbf{r}, t)\right\rangle=0
$$

guarantees the conservation of quantum numbers.

Gauge symmetry can also be broken by introducing infinitesimal sources and defining the Bogoliubov quasiaverages $[39,40]$. Both these methods of gauge symmetry breaking, by means of the Bogoliubov shift (1) and by means of infinitesimal sources, have been shown to be equivalent and sufficient for describing Bose-Einstein condensation [39-45].

The operators of observables, after the Bogoliubov shift (1), are defined on the Fock space $\mathcal{F}\left(\psi_{1}\right)$ generated by $\psi_{1}^{\dagger}$ (see mathematical details in Refs. [23,46]). The energy operator is taken in the standard form

$$
\begin{aligned}
\hat{H} & =\int \hat{\psi}^{\dagger}(\mathbf{r})\left(-\frac{\nabla^{2}}{2 m}+U\right) \hat{\psi}(\mathbf{r}) d \mathbf{r} \\
& +\frac{1}{2} \int \hat{\psi}^{\dagger}(\mathbf{r}) \hat{\psi}^{\dagger}\left(\mathbf{r}^{\prime}\right) \Phi\left(\mathbf{r}-\mathbf{r}^{\prime}\right) \hat{\psi}\left(\mathbf{r}^{\prime}\right) \hat{\psi}(\mathbf{r}) d \mathbf{r} d \mathbf{r}^{\prime},
\end{aligned}
$$

in which, for breavity, the time dependence of the field operators is not shown explicitly; $U=U(\mathbf{r}, t)$ is an external field, if any; and $\Phi(\mathbf{r})$ is the interaction potential.

In what follows, we shall consider a uniform system, with no external fields, $U=0$. The system is assumed to be equilibrium. An equilibrium statistical system is to be characterized by a representative statistical ensemble [47], which is the couple $\left\{\mathcal{F}\left(\psi_{1}\right), \hat{\rho}\right\}$ of the Fock space $\mathcal{F}\left(\psi_{1}\right)$ and the appropriate statistical operator $\hat{\rho}$. The latter can be found from the minimization of the information functional $[46,47]$, incorporating normalization conditions (3) and (4). This yields [21-26] the statistical operator

$$
\hat{\rho}=\frac{e^{-\beta H\left[\eta, \psi_{1}\right]}}{\operatorname{Tr} e^{-\beta H\left[\eta, \psi_{1}\right]}},
$$

in which $\beta=1 / T$ is inverse temperature and the grand Hamiltonian is

$$
H\left[\eta, \psi_{1}\right]=\hat{H}-\mu_{0} N_{0}-\mu_{1} \hat{N}_{1},
$$

where $\mu_{0}$ and $\mu_{1}$ are the Lagrange multipliers guaranteeing the validity of normalization conditions (3) and (4).

For a uniform system, we may set

$$
\eta(\mathbf{r}, t)=\sqrt{\rho_{0}} \quad\left(\rho_{0} \equiv \frac{N_{0}}{V}\right),
$$

with $V$ being the system volume. From $\Phi(\mathbf{r})$ and $\psi_{1}(\mathbf{r})$, we pass to their Fourier transforms $\Phi_{\mathbf{k}}$ and $a_{\mathbf{k}}$, respectively. The lowest nonzero averages of the products of $a_{\mathbf{k}}$ are the normal average

$$
n_{\mathbf{k}} \equiv\left\langle a_{\mathbf{k}}^{\dagger} a_{\mathbf{k}}\right\rangle
$$

and the anomalous average

$$
\sigma_{\mathbf{k}} \equiv\left\langle a_{\mathbf{k}} a_{-\mathbf{k}}\right\rangle
$$

The average $\left\langle a_{\mathbf{k}}\right\rangle=0$ is zero, according to condition (6).

The higher orders of the products of $a_{\mathbf{k}}$ can be simplified in the Hartree-Fock-Bogoliubov approximation [22-26]. To this end, we define

$$
\omega_{\mathbf{k}} \equiv \frac{k^{2}}{2 m}+\rho \Phi_{0}+\rho_{0} \Phi_{\mathbf{k}}+\frac{1}{V} \sum_{\mathbf{p} \neq 0} n_{\mathbf{p}} \Phi_{\mathbf{k}+\mathbf{p}}-\mu_{1}
$$

and

$$
\Delta_{\mathbf{k}} \equiv \rho_{0} \Phi_{\mathbf{k}}+\frac{1}{V} \sum_{\mathbf{p} \neq 0} \sigma_{\mathbf{p}} \Phi_{\mathbf{k}+\mathbf{p}}
$$

Then the grand Hamiltonian (9) acquires the form

$$
\begin{aligned}
H_{\mathrm{HFB}} & =E_{\mathrm{HFB}}+\sum_{\mathbf{k} \neq 0} \omega_{\mathbf{k}} a_{\mathbf{k}}^{\dagger} a_{\mathbf{k}} \\
& +\frac{1}{2} \sum_{\mathbf{k} \neq 0} \Delta_{\mathbf{k}}\left(a_{\mathbf{k}}^{\dagger} a_{-\mathbf{k}}^{\dagger}+a_{-\mathbf{k}} a_{\mathbf{k}}\right)
\end{aligned}
$$

with the nonoperator part

$$
\begin{aligned}
E_{\mathrm{HFB}} & =\left(\frac{1}{2} \rho_{0} \Phi_{0}-\mu_{0}\right) N_{0}-\frac{1}{2} \rho_{1}^{2} \Phi_{0} V \\
& -\frac{1}{2 V} \sum_{\mathbf{k}, \mathbf{p} \neq 0} \Phi_{\mathbf{k}+\mathbf{p}}\left(n_{\mathbf{k}} n_{\mathbf{p}}+\sigma_{\mathbf{k}} \sigma_{\mathbf{p}}\right),
\end{aligned}
$$

where $\rho_{1} \equiv N_{1} / V$.

The grand thermodynamic potential is

$$
\Omega=-T \ln \operatorname{Tr} e^{-\beta H\left[\eta, \psi_{1}\right]} .
$$

For thermodynamic stability, the number of condensed particles $N_{0}$ is to be a minimizer of the grand potential (17), which implies

$$
\frac{\partial \Omega}{\partial N_{0}}=\left\langle\frac{\partial H\left[\eta, \psi_{1}\right]}{\partial N_{0}}\right\rangle=0 .
$$

The latter defines the condensate chemical potential

$$
\mu_{0}=\rho \Phi_{0}+\frac{1}{V} \sum_{\mathbf{p} \neq 0}\left(n_{\mathbf{p}}+\sigma_{\mathbf{p}}\right) \Phi_{\mathbf{p}}
$$

The grand Hamiltonian (15) can be diagonalized by means of the Bogoliubov canonical transformation

$$
\begin{aligned}
& a_{\mathbf{k}}=u_{\mathbf{k}} b_{\mathbf{k}}+v_{-\mathbf{k}}^{*} b_{-\mathbf{k}}^{\dagger}, \quad u_{\mathbf{k}}^{2}=\frac{\omega_{\mathbf{k}}+\varepsilon_{\mathbf{k}}}{2 \varepsilon_{\mathbf{k}}}, \\
& v_{\mathbf{k}}^{2}=\frac{\omega_{\mathbf{k}}-\varepsilon_{\mathbf{k}}}{2 \varepsilon_{\mathbf{k}}}
\end{aligned}
$$

with the Bogoliubov spectrum

$$
\varepsilon_{\mathbf{k}}=\sqrt{\omega_{\mathbf{k}}^{2}-\Delta_{\mathbf{k}}^{2}}
$$

Then Hamiltonian (15) reduces to the diagonal form 


$$
H_{\mathrm{B}}=E_{\mathrm{B}}+\sum_{\mathbf{k} \neq 0} \varepsilon_{\mathbf{k}} b_{\mathbf{k}}^{\dagger} b_{\mathbf{k}}
$$

in which

$$
E_{\mathrm{B}}=E_{\mathrm{HFB}}+\frac{1}{2} \sum_{\mathbf{k} \neq 0}\left(\varepsilon_{\mathbf{k}}-\omega_{\mathbf{k}}\right) .
$$

The existence of Bose-Einstein condensate requires the condition

$$
\lim _{k \rightarrow 0} \varepsilon_{\mathbf{k}}=0, \quad \varepsilon_{\mathbf{k}} \geq 0
$$

from which the chemical potential of uncondensed particles follows as

$$
\mu_{1}=\rho \Phi_{0}+\frac{1}{V} \sum_{\mathbf{p} \neq 0}\left(n_{\mathbf{p}}-\sigma_{\mathbf{p}}\right) \Phi_{\mathbf{p}} .
$$

The Lagrange multipliers (19) and (25) are, evidently, different. They would coincide only for the ideal gas, when $\Phi_{\mathbf{k}} \equiv 0$, hence $\mu_{0}=\mu_{1}=0$. But for a nonideal system, $\mu_{0} \neq \mu_{1}$. Sometimes, one sets these potentials equal by omitting the anomalous average $\sigma_{\mathbf{p}}$, which corresponds to the Shohno model [48]. However, direct calculations $[25,26,49]$ demonstrate that the anomalous average is of the order and can even be larger than the normal average. Therefore, omitting the larger quantity than what is retained has nothing to do with a reasonable approximation. In addition, neglecting the anomalous average makes the system unstable [21,50,51].

\section{STRUCTURE FACTOR}

The structure factor is an important characteristic, which can be measured in scattering experiments, such as neutron or photon scatterings [52]. Let us consider, for concreteness, the neutron scattering. A neutron, with the initial energy $E_{i}$ and momentum $\mathbf{k}_{i}$, scatters on an atom, acquiring the final energy $E_{f}$ and momentum $\mathbf{k}_{f}$. The momentum and energy, transferred by a neutron to an atom, are $\mathbf{k} \equiv \mathbf{k}_{i}-\mathbf{k}_{f}$ and $\omega \equiv E_{i}-E_{f}$. The doubledifferential scattering cross section of neutrons on the system can be written [38] as

$$
\frac{1}{N} \frac{d^{2} \sigma}{d \Omega_{\mathbf{k}} d \omega}=b_{s}^{2} \frac{k_{f}}{k_{i}} S_{\text {tot }}(\mathbf{k}, \omega),
$$

where $\Omega_{\mathbf{k}}$ is a solid angle around $\mathbf{k} ; b_{s}$ is the scattering length of the neutron on an atom; and $S_{\text {tot }}(\mathbf{k}, \omega)$ is the total dynamic structure factor. The latter is defined by the expression

$$
\begin{aligned}
& S_{\text {tot }}(\mathbf{k}, \omega)=\frac{1}{N} \\
& \times \int R\left(\mathbf{r}, t, \mathbf{r}^{\prime}, 0\right) \exp \left\{-i \mathbf{k}\left(\mathbf{r}-\mathbf{r}^{\prime}\right)+i \omega t\right\} d \mathbf{r} d \mathbf{r}^{\prime} d t,
\end{aligned}
$$

in which

$$
R\left(\mathbf{r}, t, \mathbf{r}^{\prime}, t^{\prime}\right) \equiv\left\langle\hat{n}(\mathbf{r}, t) \hat{n}\left(\mathbf{r}^{\prime}, t^{\prime}\right)\right\rangle
$$

is the density-density correlation function, and the density operator is

$$
\hat{n}(\mathbf{r}, t) \equiv \hat{\psi}^{\dagger}(\mathbf{r}, t) \hat{\psi}(\mathbf{r}, t) .
$$

The total dynamic structure factor (27) can be decomposed into two terms

$$
S_{\mathrm{tot}}(\mathbf{k}, \omega)=S_{e}(\mathbf{k}, \omega)+S(\mathbf{k}, \omega)
$$

the first term

$$
S_{e}(\mathbf{k}, \omega) \equiv \frac{1}{N} \int \rho(\mathbf{r}, t) \rho\left(\mathbf{r}^{\prime}, 0\right) \exp \left\{-i \mathbf{k}\left(\mathbf{r}-\mathbf{r}^{\prime}\right)+i \omega t\right\} d \mathbf{r} d \mathbf{r}^{\prime} d t
$$

with $\rho(\mathbf{r}, t) \equiv\langle\hat{n}(\mathbf{r}, t)\rangle$, is responsible for elastic scattering and is called the disconnected dynamic structure factor; the second term, being called connected,

$$
S(\mathbf{k}, \omega) \equiv \frac{1}{N} \int\left[R\left(\mathbf{r}, t, \mathbf{r}^{\prime}, 0\right)-\rho(\mathbf{r}, t) \rho\left(\mathbf{r}^{\prime}, 0\right)\right] \exp \left\{-i \mathbf{k}\left(\mathbf{r}-\mathbf{r}^{\prime}\right)+i \omega t\right\} d \mathbf{r} d \mathbf{r}^{\prime} d t
$$

describes inelastic scattering.

The elastic dynamic factor (31) is nontrivial for nonuniform systems [53,54], but for a uniform equilibrium system, when $\rho(\mathbf{r}, t)=\rho$, it is very simple, being

$$
S_{e}(\mathbf{k}, \omega)=(2 \pi)^{4} \rho \delta(\mathbf{k}) \delta(\omega) .
$$

Therefore, for uniform systems, more attention is payed to the inelastic dynamic factor (32). The latter enjoys the sum rules, one of which

$$
\int_{-\infty}^{\infty} S(\mathbf{k}, \omega) \frac{d \omega}{2 \pi}=S(\mathbf{k})
$$

defines the static structure factor $S(\mathbf{k})$, another gives

$$
\int_{-\infty}^{\infty} \omega S(\mathbf{k}, \omega) \frac{d \omega}{2 \pi}=\frac{k^{2}}{2 m}
$$

and the third, connects the integral 


$$
\int_{-\infty}^{\infty} \frac{1}{\omega} S(\mathbf{k}, \omega) \frac{d \omega}{2 \pi}=-\frac{\operatorname{Re} \chi(\mathbf{k}, 0)}{2 \rho},
$$

with the responce function $\chi(\mathbf{k}, \omega)$. At very large momentum $\mathbf{k} \rightarrow \infty$, there are the following asymptotic equalities:

$$
\begin{aligned}
& \int_{-\infty}^{\infty} S(\mathbf{k}, \omega) \frac{d \omega}{2 \pi} \simeq 1 \quad(k \rightarrow \infty), \\
& \int_{-\infty}^{\infty}\left(\omega-\frac{k^{2}}{2 m}\right) S(\mathbf{k}, \omega) \frac{d \omega}{2 \pi} \simeq 0, \\
& \int_{-\infty}^{\infty}\left(\omega-\frac{k^{2}}{2 m}\right)^{2} S(\mathbf{k}, \omega) \frac{d \omega}{2 \pi} \simeq \frac{2 k^{2}}{3 m} \frac{\langle\hat{K}\rangle}{N},
\end{aligned}
$$

$$
\int_{-\infty}^{\infty}\left(\omega-\frac{k^{2}}{2 m}\right)^{3} S(\mathbf{k}, \omega) \frac{d \omega}{2 \pi} \simeq 0
$$

here $\langle\hat{K}\rangle$ is the average kinetic energy of the system.

For an equilibrium system, where

$$
\rho(\mathbf{r}, t)=\rho(\mathbf{r})=\langle\hat{n}(\mathbf{r})\rangle
$$

with $\hat{n}(\mathbf{r}) \equiv \hat{n}(\mathbf{r}, 0)$, the static structure factor, defined in Eq. (34), becomes

$$
S(\mathbf{k})=\frac{1}{N} \int\left[\left\langle\hat{n}(\mathbf{r}) \hat{n}\left(\mathbf{r}^{\prime}\right)\right\rangle-\rho(\mathbf{r}) \rho\left(\mathbf{r}^{\prime}\right)\right] \exp \left\{-i k\left(\mathbf{r}-\mathbf{r}^{\prime}\right)\right\} d \mathbf{r} d \mathbf{r}^{\prime}
$$

Let us introduce the Fourier transform of the density operator (29),

$\hat{\rho}_{\mathbf{k}} \equiv \int \hat{n}(\mathbf{r}) e^{-i \mathbf{k} \cdot \mathbf{r}} d \mathbf{r}, \quad \hat{n}(\mathbf{r})=\frac{1}{V} \sum_{\mathbf{k}} \hat{\rho}_{\mathbf{k}} e^{i \mathbf{k} \cdot \mathbf{r}}$.

The following properties hold:

$$
\begin{aligned}
& {\left[\hat{\rho}_{\mathbf{k}}, \hat{\rho}_{\mathbf{p}}\right]=0, \quad\left[\hat{n}(\mathbf{r}), \hat{n}\left(\mathbf{r}^{\prime}\right)\right]=0,} \\
& \hat{\rho}_{\mathbf{k}}^{+}=\hat{\rho}_{-\mathbf{k}}, \quad \hat{n}^{+}(\mathbf{r})=n(\mathbf{r}) .
\end{aligned}
$$

The zero component of $\rho_{\mathbf{k}}$ yields the total number of particles,

$$
\hat{\rho}_{0}=\int \hat{n}(\mathbf{r}) d \mathbf{r}=\hat{N} .
$$

The static structure factor (38) can be expressed as

$$
S(\mathbf{k})=\frac{\Delta^{2}\left(\hat{\rho}_{\mathbf{k}}\right)}{N}
$$

through the dispersion

$$
\Delta^{2}\left(\hat{\rho}_{\mathbf{k}}\right) \equiv\left\langle\hat{\rho}_{\mathbf{k}}^{+} \hat{\rho}_{\mathbf{k}}\right\rangle-\left\langle\hat{\rho}_{\mathbf{k}}^{+}\right\rangle\left\langle\hat{\rho}_{\mathbf{k}}\right\rangle .
$$

The central value of the structure factor (41),

$$
S(0)=\frac{\Delta^{2}(\hat{N})}{N}=\rho T \kappa_{T}=\frac{T}{m s^{2}}
$$

connects it with the particle dispersion $\Delta^{2}(\hat{N})=\Delta^{2}\left(\hat{\rho}_{0}\right)$, the isothermal compressibility

$$
\kappa_{T} \equiv-\frac{1}{V}\left(\frac{\partial V}{\partial P}\right)_{T N}=\frac{1}{\rho}\left(\frac{\partial \rho}{\partial P}\right)_{T N},
$$

where $P$ is pressure, and with the hydrodynamic sound velocity $s$,

$$
s^{2} \equiv \frac{1}{m}\left(\frac{\partial P}{\partial \rho}\right)_{T}=\frac{1}{m \rho \kappa_{T}} .
$$

Thus, the isothermal compressibility and sound velocity are expressed through the structure factor as

$$
\kappa_{T}=\frac{S(0)}{\rho T}, \quad s^{2}=\frac{T}{m S(0)} .
$$

Relations (43) and (46) are exact, being valid for any equilibrium, not necessarily uniform, system.

Passing to a uniform system, for the Bogoliubov shift (1), we have

$$
\hat{\psi}(\mathbf{r})=\sqrt{\rho_{0}}+\psi_{1}(\mathbf{r}) .
$$

The density operator (29) becomes

$$
\hat{n}(\mathbf{r})=\rho_{0}+\hat{n}_{1}(\mathbf{r})+\sqrt{\rho_{0}}\left[\psi_{1}^{\dagger}(\mathbf{r})+\psi_{1}(\mathbf{r})\right],
$$

where the density operator of uncondensed particles is

$$
\hat{n}_{1}(\mathbf{r}) \equiv \psi_{1}^{\dagger}(\mathbf{r}) \psi_{1}(\mathbf{r}) .
$$

The Fourier transform of the total density operator (48) takes the form

$$
\hat{\rho}_{\mathbf{k}}=\delta_{\mathbf{k} 0} N_{0}+\sqrt{N}\left(a_{-\mathbf{k}}^{\dagger}+a_{\mathbf{k}}\right)+\sum_{\mathbf{p} \neq 0} a_{\mathbf{k}+\mathbf{p}}^{\dagger} a_{\mathbf{p}} .
$$

Because of the properties

$$
\left\langle a_{\mathbf{k}}^{\dagger} a_{\mathbf{p}}\right\rangle=\delta_{\mathbf{k p}} n_{\mathbf{k}}, \quad\left\langle a_{\mathbf{k}}\right\rangle=0,
$$

we have

$$
\left\langle\hat{\rho}_{\mathbf{k}}\right\rangle=\delta_{\mathbf{k} 0} N .
$$

For dispersion (42), we find 


$$
\begin{aligned}
\Delta^{2}\left(\hat{\rho}_{\mathbf{k}}\right) & =N+2 N_{0}\left(n_{\mathbf{k}}+\sigma_{\mathbf{k}}\right) \\
& +\sum_{\mathbf{p}, \mathbf{q} \neq 0}\left\langle a_{\mathbf{p}}^{\dagger} a_{\mathbf{k}+\mathbf{q}}^{\dagger} a_{\mathbf{k}+\mathbf{p}} a_{\mathbf{q}}\right\rangle-\delta_{\mathbf{k} 0} N_{1}^{2} .
\end{aligned}
$$

When the interaction potential $\Phi(\mathbf{r})$ is singular at $r \rightarrow 0$, then pair atomic correlations are very important $[52,55]$. But here, we have assumed that the interaction potential is soft, so it possesses the Fourier transform $\Phi_{\mathbf{k}}$. For the soft potential, we can apply the HartreeFock-Bogoliubov (HFB) approximation. Employing the latter, one has to be cautious, since its blind application can lead to unphysical results. Thus, if we formally decoupled the four-operator term in dispersion (52), by using the HFB approximation, we would come to

$$
\begin{aligned}
\Delta^{2}\left(\hat{\rho}_{\mathbf{k}}\right) \longrightarrow N & +2 N_{0}\left(n_{\mathbf{k}}+\sigma_{\mathbf{k}}\right) \\
& +\sum_{\mathbf{p} \neq 0}\left(n_{\mathbf{k}+\mathbf{p}} n_{\mathbf{p}}+\sigma_{\mathbf{k}+\mathbf{p}} \sigma_{\mathbf{p}}\right) .
\end{aligned}
$$

However, one should keep in mind that the use of the HFB approximation results in the Hamiltonian (22), which is quadratic with respect to the operators of uncondensed particles. Therefore, all expressions of the order higher than two with respect to these operators are not well defined in this approximation. One could consider such high-order terms only if their behavior were reasonable. But sometimes these higher-order terms result in unphysical divergencies, which simply means that they should be omitted, being outside of the region of applicability of the used approximation. In the second-order approximation, only the second-order terms are well defined $[14,21,23,50,51]$. Consequently, dispersion (52), in the HFB approximation, has to be written as

$$
\Delta^{2}\left(\hat{\rho}_{\mathbf{k}}\right)=N+2 N\left(n_{\mathbf{k}}+\sigma_{\mathbf{k}}\right) .
$$

Recall that the normal and anomalous averages in the HFB approximation [23-26] are

$$
\begin{aligned}
n_{\mathbf{k}} & =\frac{\omega_{\mathbf{k}}}{2 \varepsilon_{\mathbf{k}}} \operatorname{coth}\left(\frac{\varepsilon_{\mathbf{k}}}{2 T}\right)-\frac{1}{2}, \\
\sigma_{\mathbf{k}} & =-\frac{\Delta_{\mathbf{k}}}{2 \varepsilon_{\mathbf{k}}} \operatorname{coth}\left(\frac{\varepsilon_{\mathbf{k}}}{2 T}\right),
\end{aligned}
$$

where $\omega_{\mathbf{k}}, \Delta_{\mathbf{k}}$, and $\varepsilon_{\mathbf{k}}$ are given by Eqs. (13), (14), and (21), respectively. The Bogoliubov spectrum (21), according to condition (24), is gapless, having the phonon form $\varepsilon_{\mathbf{k}} \simeq c k$ at $k \rightarrow 0$, with the sound velocity $c$ defined by the equation

$$
m c^{2}=\rho_{0} \Phi_{0}+\frac{1}{V} \sum_{\mathbf{p} \neq 0} \sigma_{\mathbf{p}} \Phi_{\mathbf{p}} .
$$

Dispersion (54) is finite. For instance, in the case of $k \rightarrow 0$, when $\Delta^{2}\left(\hat{\rho}_{0}\right)=\Delta^{2}(\hat{N})$, we have

$$
\Delta^{2}(\hat{N})=N\left[1+2 \lim _{k \rightarrow 0}\left(n_{\mathbf{k}}+\sigma_{\mathbf{k}}\right)\right] .
$$

Using Eqs. (55), we get

$$
\lim _{k \rightarrow 0}\left(n_{\mathbf{k}}+\sigma_{\mathbf{k}}\right)=\frac{1}{2}\left(\frac{T}{m c^{2}}-1\right) .
$$

Therefore,

$$
\Delta^{2}(\hat{N})=\frac{N T}{m c^{2}}
$$

Consequently,

$$
S(0)=\frac{T}{m c^{2}}, \quad \kappa_{T}=\frac{1}{\rho m c^{2}}, \quad s=c .
$$

The structure factor (41), according to Eq. (54), takes the form

$$
S(\mathbf{k})=1+2\left(n_{\mathbf{k}}+\sigma_{\mathbf{k}}\right) .
$$

Substituting here expressions (55), we find

$$
S(\mathbf{k})=\frac{k^{2}}{2 m \varepsilon_{\mathbf{k}}} \operatorname{coth}\left(\frac{\varepsilon_{\mathbf{k}}}{2 T}\right) .
$$

Note that the Feynman relation

$$
\varepsilon_{\mathbf{k}}=\frac{k^{2}}{2 m S(\mathbf{k})} \quad(T=0)
$$

is valid only at zero temperature. The asymptotic properties of the structure factor (60) are:

$$
\begin{aligned}
& S(\mathbf{k}) \simeq \frac{T}{m c^{2}} \quad(k \rightarrow 0) \\
& S(\mathbf{k}) \simeq 1 \quad(k \rightarrow \infty) .
\end{aligned}
$$

If instead of expression (54), one would use Eq. (53), then the structure factor $S(\mathbf{k})$ would be divergent, which has no physical sense. Hence, Eq. (54) provides the correct mean-field approximation. The correctness of Eq. (54) will be analyzed in more detail in the following section.

\section{APPROXIMATION ANALYSIS}

The form of the structure factor (60) is due to that of dispersion (54). Here, we shall analyze the correctness of the approximation in which Eq. (53) has been replaced by Eq. (54).

We shall also stress the necessity of correctly taking into account the anomalous averages and the importance of gauge symmetry breaking. 


\section{A. Preservation of approximation order}

The replacement of Eq. (53) by Eq. (54) is based on the requirement of preserving the approximation order with respect to the operators of uncondensed particles. Suppose that we would go outside of the region of applicability of the considered second-order approximation and would retain the last term in Eq. (53). Then $\Delta^{2}\left(\hat{\rho}_{\mathbf{k}}\right)$ would be proportional to $N^{4 / 3}$, hence $S(\mathbf{k})$ proportional to $N^{1 / 3}$, that is, the structural factor $S(\mathbf{k})$ would be divergent, hence, unphysical. To show this, consider $\Delta^{2}\left(\hat{\rho}_{0}\right)=\Delta^{2}(\hat{N})$. Then the last terms in Eq. (53) give

$$
\begin{gathered}
V \int n_{\mathbf{k}}^{2} \frac{d \mathbf{k}}{(2 \pi)^{3}} \sim \frac{(m T)^{2}}{2 \pi^{2}} V^{4 / 3}, \\
V \int \sigma_{\mathbf{k}}^{2} \frac{d \mathbf{k}}{(2 \pi)^{3}} \sim \frac{(m T)^{2}}{2 \pi^{2}} V^{4 / 3} .
\end{gathered}
$$

Therefore,

$$
\Delta^{2}(\hat{N}) \sim \frac{(m T)^{2}}{\pi} V^{4 / 3}
$$

In this way, when $T>0$, in the thermodynamic limit

$$
\begin{gathered}
S(0) \propto N^{1 / 3} \rightarrow \infty, \quad s \propto N^{-1 / 6} \rightarrow 0, \\
\kappa_{T} \propto N^{1 / 3} \rightarrow \infty \quad(N \rightarrow \infty) .
\end{gathered}
$$

This means an absolute instability of the system at any finite temperature.

At zero temperature, Eqs. (55) reduce to

$$
\begin{aligned}
& n_{\mathbf{k}}=\frac{1+2 q^{2}}{4 q \sqrt{1+q^{2}}}-\frac{1}{2}, \\
& \sigma_{\mathbf{k}}=-\frac{1}{4 q \sqrt{1+q^{2}}} \quad\left(q \equiv \frac{k}{2 m c}, T=0\right) .
\end{aligned}
$$

From here,

$$
\lim _{k \rightarrow 0}\left(n_{\mathbf{k}}+\sigma_{\mathbf{k}}\right)=-\frac{1}{2} \quad(T=0) .
$$

The last terms in Eq. (53), at $T=0$, are finite, since

$$
\int n_{\mathbf{k}}^{2} \frac{d \mathbf{k}}{(2 \pi)^{3}}<\infty, \quad \int \sigma_{\mathbf{k}}^{2} \frac{d \mathbf{k}}{(2 \pi)^{3}}<\infty .
$$

The dispersion $\Delta^{2}(\hat{N}) \propto N$ is thermodynamically normal. The value $S(0) \propto$ const $>0$ is finite. However, as follows from relations (46),

$$
\kappa_{T} \propto \frac{1}{T} \rightarrow \infty, \quad s \propto T \rightarrow 0 \quad(T \rightarrow 0),
$$

which tells that the system is again unstable. Thus, at any temperature $T \geq 0$, in the thermodynamic limit, one has $s=0$ and $\kappa_{T}=\infty$. Hence, such a system would always be unstable. But, of course, the appearance of this instability is not physical and is caused only by going outside of the region of applicability of the used approximation. Preserving the approximation order requires to reject the last terms in Eq. (53). Then no unphysical divergencies appear.

\section{B. Importance of anomalous averages}

One of the popular tricks when considering Bosecondensed systems is the omission of the anomalous average $\sigma_{\mathbf{k}}$, which corresponds to the Shohno model [48]. One often ascribes this unjustified trick to Popov. But, it is apparent from the original works of Popov [56,57], which are cited in this respect, he has never suggested this trick. Here we show that omitting the anomalous average is principally wrong, since then the system becomes unstable and its structural factor infinite, which is, certainly, of no sense.

Assume that the anomalous average $\sigma_{\mathbf{k}}$ in Eq. (54) is set to zero. Then we have

$$
\Delta^{2}(\hat{N}) \rightarrow N\left(1+2 \lim _{k \rightarrow 0} n_{\mathbf{k}}\right) .
$$

At nonzero temperature,

$$
n_{\mathbf{k}} \simeq \frac{m T}{k^{2}} \quad(T>0, k \rightarrow 0) .
$$

Since $k \sim 1 / V^{1 / 3}$, we get

$$
\Delta^{2}(\hat{N}) \sim \frac{2 m T}{\rho^{2 / 3}} N^{5 / 2} \quad(T>0) .
$$

Then, at finite temperature,

$$
S(0) \sim \frac{2 m T}{\rho^{2 / 3}} N^{2 / 3}, \quad \kappa_{T} \sim \frac{2 m}{\rho^{5 / 3}} N^{2 / 3} .
$$

These quantities diverge in the thermodynamic limit, which implies instability.

At zero temperature,

$$
n_{\mathbf{k}} \simeq \frac{m c}{2 k} \quad(T=0, k \rightarrow 0) .
$$

From here

$$
\Delta^{2}(\hat{N}) \sim \frac{m c}{\rho^{1 / 3}} N^{4 / 3} \quad(T=0) .
$$

Hence, as $T \rightarrow 0$, we have

$$
S(0) \sim \frac{m c}{\rho^{1 / 3}} N^{1 / 3}, \quad \kappa_{T} \sim \frac{m c}{\rho^{4 / 3} T} N^{1 / 3} .
$$

The structural factor diverges in the thermodynamic limit, and the isothermal compressibility $\kappa_{T}$ becomes divergent even at finite $N$, when $T \rightarrow 0$.

Thus, at all temperatures $T \geq 0$, we come to the senseless unphysical behavior, when

$$
S(\mathbf{k}) \rightarrow \infty, \quad s \rightarrow 0, \quad \kappa_{T} \rightarrow \infty
$$

This proves that the anomalous average plays a principally important role and cannot be omitted. 


\section{Gauge symmetry breaking}

Gauge symmetry breaking is known to be sufficient for describing Bose-Einstein condensation. This follows from the general theorems, independent of approximations, proved by Bogoliubov [39,40], Ginibre [41], and Lieb et al. [42-45]. At the same time, in the PenroseOnsager [58] scheme of Bose condensation no explicit breaking of symmetry is required, but the appearance of condensate is manifested in the properties of the densitymatrices eigenvalues or their order indices [59,60]. Here we give arguments that, in the frame of a mean-field approximation, gauge symmetry breaking is necessary.

Suppose, we consider a mean-field approximation without breaking the gauge symmetry. Then the numberof-particle dispersion has the standard form

$$
\Delta^{2}(\hat{N})=\sum_{\mathbf{k}} n_{\mathbf{k}}\left(1+n_{\mathbf{k}}\right)=\Delta^{2}\left(\hat{N}_{0}\right)+\Delta^{2}\left(\hat{N}_{1}\right)
$$

which can be separated into two terms,

$$
\Delta^{2}\left(\hat{N}_{0}\right)=N_{0}\left(1+N_{0}\right), \quad \Delta^{2}\left(\hat{N}_{1}\right)=\sum_{\mathbf{k} \neq 0} n_{\mathbf{k}}\left(1+n_{\mathbf{k}}\right)
$$

corresponding to condensed and uncondensed particles, respectively. The occurrence of Bose-Einstein condensation implies that the number of condensed particles becomes proportional to the total number of particles, $N_{0} \propto N$. As a result,

$$
\Delta^{2}\left(\hat{N}_{0}\right) \propto N^{2} \quad\left(N_{0} \propto N\right) .
$$

Consequently, $\Delta^{2}(\hat{N}) \propto N^{2}$. Therefore, the isothermal compressibility and the structure factor diverge in the thermodynamic limit as

$$
S(\mathbf{k}) \propto N \rightarrow \infty, \quad \kappa_{T} \propto N \rightarrow \infty
$$

which means instability.

In this way, the use of the mean-field approximation requires that gauge symmetry be broken. It may be that the exact consideration or some refined approximations (see, e. g., Ref. [17]) allow for a description without gauge symmetry breaking. However, for treating Bose-Einstein condensation in a mean-field picture, gauge symmetry breaking is the necessary and sufficient condition. This also concerns any perturbation theory starting with a mean-field approximation.

\section{CONCLUSION}

A Bose system with Bose-Einstein condensate is considered. The properties of the structure factor are investigated in the frame of the self-consistent mean-field approximation, which is both conserving and gapless. The completed analysis demonstrates the importance of preserving the approximation order. Going outside of the region of applicability of the used approximation can lead to the appearance of unphysical divergences. In the system with broken gauge symmetry, it is crucially important to accurately take account of anomalous averages. Omitting the latter is unjustified and results in the occurrence of fictitious instability. It is also shown that gauge symmetry breaking is necessary in any theory based on a mean-field picture.
[1] H. W. Jackson, Phys. Rev. A 10, 278 (1974).

[2] D. M. Ceperley, Rev. Mod. Phys. 67, 279 (1995).

[3] L. J. Rodriguez, H. A. Gersch, H. A. Mook, Phys. Rev. A 9, 2085 (1974).

[4] P. E. Sokol, T. R. Sosnick, W. M. Snow, in Momentum Distribution in Liquid Helium, edited by R. N. Silver, P. E. Sokol (Plenum, New York, 1989), p. 139.

[5] K. W. Herwig, P. E. Sokol, T. R. Sosnick, W. M. Snow, R. C. Blasdell, Phys. Rev. B 41, 103 (1990).

[6] I. V. Bogoyavlensky, L. P. Karnatsevich, G. A. Kozlov, A. V. Puchkov, Low Temp. Phys. 16, 139 (1990).

[7] T. R. Sosnick, W. M. Snow, R. N. Silver, P. E. Sokol, Phys. Rev. B 43, 216 (1991).

[8] I. V. Bogoyavlensky, A. V. Puchkov, A. N. Skomorokhov, S. V. Poupko, Physica B 234, 324 (1997).

[9] W. Montfrooij, E. C. Svensson, R. Verberg, R. M. Crevecoeur, I. M. de Schepper, cond-mat/0603523 (2006).

[10] L. Pitaevskii, S. Stringari, Bose-Einstein Condensation in Dilute Gases (Clarendon, Oxford, 2003).

[11] P. W. Courteille, V. S. Bagnato, V. I. Yukalov, Laser Phys. 11, 659 (2001).

[12] J. O. Andersen, Rev. Mod. Phys. 76, 599 (2004).

[13] K. Bongs, K. Sengstock, Rep. Prog. Phys. 67, 907 (2004).

[14] V. I. Yukalov, Laser Phys. Lett. 1, 435 (2004).
[15] V. I. Yukalov, M. D. Girardeau, Laser Phys. Lett. 2, 375 (2005).

[16] J. Steinhauer, R. Ozeri, N. Katz, N. Davidson, Phys. Rev. Lett. 88, 120407 (2002).

[17] I. A. Vakarchuk, Theor. Math. Phys. 23, 260 (1975).

[18] N. N. Bogoliubov, J. Phys. (Moscow) 11, 23 (1947).

[19] N. N. Bogoliubov, Moscow Univ. Phys. Bull. 7, 43 (1947).

[20] P. C. Hohenberg, P. C. Martin, Ann. Phys. 34, 291 (1965)

[21] V. I. Yukalov, Phys. Rev. E 72, 066119 (2005).

[22] V. I. Yukalov, Laser Phys. Lett. 3, 406 (2006).

[23] V. I. Yukalov, Laser Phys. 16, 511 (2006).

[24] V. I. Yukalov, Phys. Lett. A 359, 712 (2006).

[25] V. I. Yukalov, H. Kleinert, Phys. Rev. A 73, 063612 (2006).

[26] V. I. Yukalov, E. P. Yukalova, Phys. Rev. A 74, 063623 (2006).

[27] E. A. Donley, N. R. Claussen, S. T. Thompson, C. E. Wieman, Nature 417, 529 (2002).

[28] C. Chin, A. J. Kerman, V. Vuletic, S. Chu, Phys. Rev. Lett. 90, 033201 (2003).

[29] J. Herbig, T. Kraemer, M. Mark, T. Weber, C. Chin, H. C. Nägerl, R. Grimm, Science 301, 1510 (2003). 
[30] S. Dürr, T. Volz, A. Marte, G. Rempe, Phys. Rev. Lett. 92, 020406 (2004).

[31] C. A. Regal, T. Ticknor, J. L. Bohn, D. S. Jin, Nature 424, 47 (2003).

[32] J. Cubizolles, T. Bourdel, S. J. Kokkelmans, G. V. Shlyapnikov, C. Salomon, Phys. Rev. Lett. 91, 240401 (2003).

[33] S. Jochim, M. Bartenstein, A. Altmeyer, G. Hendl, C. Chin, J. H. Denschlag, R. Grimm, Phys. Rev. Lett. 91, 240402 (2003).

[34] M. W. Zwierlein, C. A. Stan, C. H. Schunck, S. M. Raupach, S. Gupta, Z. Hadzibabic, W. Ketterle, Phys. Rev. Lett. 91, 250401 (2003).

[35] C. A. Regal, M. Greiner, D. S. Jin, Phys. Rev. Lett. 92, 040403 (2004).

[36] T. Köhler, T. Gasenzer, P. S. Julienne, K. Burnett, Phys. Rev. Lett. 91, 230401 (2003).

[37] V. A. Yurovsky, cond-mat/0611054 (2006).

[38] V. I. Yukalov, A. S. Shumovsky, Lectures on Phase Transitions (World Scientific, Singapore, 1990).

[39] N. N. Bogoliubov, Lectures on Quantum Statistics (Gordon and Breach, New York, 1967), Vol. 1.

[40] N. N. Bogoliubov, Lectures on Quantum Statistics (Gordon and Breach, New York, 1970), Vol. 2.

[41] J. Ginibre, Commun. Math. Phys. 8, 26 (1968).

[42] E. H. Lieb, R. Seiringer, J. Yngvason, Phys. Rev. Lett. 94, 080401 (2005).

[43] A. Süto, Phys. Rev. Lett. 94, 080402 (2005).

[44] E. H. Lieb, R. Seiringer, J. P. Solovej, J. Yngvason,
The Mathematics of the Bose Gas and its Condensation (Birkhauser, Basel, 2005).

[45] E. H. Lieb, R. Seiringer, J. Yngvason, cond-mat/0610034 (2006).

[46] V. I. Yukalov, Statistical Green's Functions (Queen's University, Kingston, 1998).

[47] V. I. Yukalov, Phys. Rep. 208, 395 (1991).

[48] N. Shohno, Prog. Theor. Phys. 31, 553 (1964).

[49] V. I. Yukalov, E. P. Yukalova, Laser Phys. Lett. 2, 506 (2005).

[50] V. I. Yukalov, Laser Phys. Lett. 2, 156 (2005).

[51] V. I. Yukalov, Phys. Lett. A 340, 369 (2005).

[52] C. D. Mahan, Many-Particle Physics (Plenum, New York, 1981).

[53] D. Belitz, A. Gold, W. Götze, J. Metzger, Phys. Rev. B 27, 4559 (1983).

[54] M. L. Rosinberg, G. Tarjus, G. Stell, J. Chem. Phys. 100, 5172 (1994).

[55] F. W. Cummings, G. F. Hyland, G. Rowlands, Phys. Lett. A 86, 370 (1981).

[56] V. N. Popov, Functional Integrals in Quantum Field Theory and Statistical Physics (Reidel, Dordrecht, 1983).

[57] V. N. Popov, Functional Integrals and Collective Modes (Cambridge University, New York, 1987).

[58] O. Penrose, L. Onsager, Phys. Rev. 104, 576 (1956).

[59] A. J. Coleman, V. I. Yukalov, Reduced Density Matrices (Springer, Berlin, 2000).

[60] V. I. Yukalov, Physica A 310, 413 (2002).

\title{
СТРУКТУРНИЙ ФАКТОР БОЗЕ-КОНДЕНСОВАНОЇ СИСТЕМИ
}

\author{
В. І. Юкалов \\ Лабораторія теоретичної фізики ім. М. Боголюбова \\ Об'єднаний інститут ядерних досліджень, Дубна, 141980, Росія
}

\begin{abstract}
Розглянуто структурний фактор бозе-системи з конденсатом Бозе-Айнштайна в межах самоузгодженого наближення середнього поля. Проведений аналіз показує принципову важливість таких трьох чинників: необхідність збереження порядку наближення, доконечність урахування аномальних середніх і необхідність порушення калібрувальної симетрії. Якщо хоча б одна з цих доконечних умов не задовольняється, то обчислення приводить до появи нефізичних розбіжностей структурного фактора, що означає виникнення фіктивної нестабільности, а це суперечить експериментам.
\end{abstract}

